\title{
MISSING DATA IMPUTATION ON IOT SENSOR NETWORKS: IMPLICATIONS FOR ON-SITE SENSOR CALIBRATION
}

This paper was downloaded from TechRxiv (https://www.techrxiv.org).

\section{LICENSE}

CC BY 4.0

SUBMISSION DATE / POSTED DATE

23-01-2021 / 26-01-2021

CITATION

Okafor, Nwamaka (2021): MISSING DATA IMPUTATION ON IOT SENSOR NETWORKS: IMPLICATIONS FOR ON-SITE SENSOR CALIBRATION. TechRxiv. Preprint. https://doi.org/10.36227/techrxiv.13633529.v1

$\mathrm{DOI}$

10.36227/techrxiv.13633529.v1 


\title{
MISSING DATA IMPUTATION ON IOT SENSOR NETWORKS: IMPLICATIONS FOR ON-SITE SENSOR CALIBRATION
}

\author{
Nwamaka U. Okafor ${ }^{\mathrm{a}}$, Declan T. Delaney \\ ${ }^{a}$ School of Electrical and Electronic Engineering, University College Dublin, Ireland
}

\begin{abstract}
IoT sensors are gaining more popularity in the environmental monitoring space due to their relatively small size, cost of acquisition and ease of installation and operation. They are becoming increasingly important supplement to traditional monitoring systems, particularly for in-situ based monitoring. However, data collection based on IoT sensors are often plagued with missing values usually occurring as a result of sensor faults, network failures, drifts and other operational issues. Several imputation strategies have been proposed for handling missing values in various application domains. This paper examines the performance of different imputation techniques including Multiple Imputation by Chain Equations (MICE), Random forest based imputation (missForest) and K-Nearest Neighbour (KNN) for handling missing values on sensor networks deployed for the quantification of Green House Gases(GHGs). Two tasks were conducted: first, Ozone $\left(\mathrm{O}_{3}\right)$ and $\mathrm{NO}_{2} / \mathrm{O}_{3}$ concentration data collected using Aeroqual and Cairclip sensors respectively over a six months data collection period were corrupted by removing data intervals at different missing periods $(p)$ where $p \in\{1$ day, 1 week, 2 weeks, 1 month $\}$ and also at random points on the dataset at varying proportion (r) where $r \in\{5 \%, 10 \%, 30 \%, 50 \%, 70 \%\}$. The missing data were then filled using the different imputation strategies and their imputation accuracy calculated. Second, the performance of sensor calibration by different regression models including Multi Linear Regression (MLR), Decision Tree (DT), Random Forest (RF) and XGBoost (XGB) trained on the different imputed datasets were evaluated. The analysis showed the MICE technique to outperform the others in imputing the missing values on both the $\mathrm{O}_{3}$ and $\mathrm{NO}_{2} / \mathrm{O}_{3}$ datasets when missingness was introduced over periods $p$. MissForest, however, outperformed the rest when missingness was introduced as randomly occuring point errors. While the analysis demonstrated the effects of missing and imputed data on sensor calibration, experimental results showed that a simple model on the imputed dataset can achieve state of-the-art result on in-situ sensor calibration, improving the data quality of the sensor.
\end{abstract}

Keywords: Internet of Things (IoT), Sensors, Missing Data, Imputation, Calibration, Regression, XGBoost, Random Forest, Supervised Learning

\section{Introduction}

To tackle the challenges of climate change, it is necessary to have reliable and robust information on Green House Gases (GHGs) emissions which contribute to this phenomenon. Expanding the measurement networks for GHGs is vital for understanding their global emission trends and the effectiveness of emission mitigation policies, strategies and initiatives. This also makes it possible to ascertain how far emission reduction targets are being met at the local, regional and global scales[1].

Low Cost Sensors (LCS) can be used to enhance spatial and temporal resolution of data acquisition for key GHG variables to corroborate satellite and remotebased sensing approaches. LCS, however, are prone to various failures including bias, drifts, precision degradation, and loss of considerable amount of data due to operational issues[2]. Missing data is a pervasive issue which occur in most real-world datasets including medical records[3, 4], geo-informatics[5], traffic flow[6] and industrial applications[7, 8].

The European Union Data Quality Directive (EU-DQD) [9] defined the data quality objective (DQO) that a monitoring method needs to comply with to be used as indicative measurement for regulative purposes. The EU-DQD is a measure of the acceptable uncertainty for indicative measurements. The directive also defined the degree of data completeness for such monitoring method. To meet these requirements and to present LCS as suitable for adoption for this purpose, 
data completeness is essential for the sensors. In addition to this, complete data points consisting of sensor outputs and labels are essential for building reliable calibration models to ensure the collection of accurate and robust dataset by LCS.

Several methods have been proposed for handling missing data in diverse application domains. Common among these methods are downsampling which is also known as Complete Case Analysis (CCA) and imputation. The main idea behind downsampling is discarding the incomplete observations i.e. dropping missing data records. Although downsampling is a very simple method for handling missing data, it comes at the cost of losing useful information on data which may be valuable even though the data is incomplete. Applying downsampling as a means of handling missing values in any statistical analysis is mostly useful when there is a large number of samples [10] but performs poorly when the rate of missing value is high[11]. Imputation can be classified as: (i) single imputation which involves replacing all missing values on a variable with a single value say zero(i.e zero imputation) or the mean, median of the observation. (ii) Multiple Imputation (MI) which is often an iterative model-based approach[12].

State of the art multiple imputation techniques can be classified into Discriminative and Generative methods. Discriminative methods include Multiple Imputation by Chain Equations (MICE)[13], Random Forest-based Imputation (Missforest)[14] and matrix completion[15]. Generative methods consist mostly of techniques based on Deep Learning (DL) e.g Denoising Autoencoders (DAE) [2][16] and Generative Adversarial Networks(GAN)[17][18]. GAN learns the latent distribution of a dataset and can generate real samples from a random noise. This technique has been successfully applied in sentence generation and face completion[19][20], Although both DAE and GAN have performed well in principle, both methods require the availability of complete dataset for training, this, however is not easily obtainable in LCS networks deployed for environmental monitoring which are often marred by missing values.

In this paper, we analysed the performance of different state of the art imputation techniques including MICE, MissForest and K-Nearest Neighbour (KNN) for handling missing values in LCS networks where values could be missing over certain period of time or at random points in time. We also evaluated the impact of missing and imputed data on the calibration of LCS using different regression models including Multi Linear Regression (MLR), Decision Tree (DT), Random Forest (RF) and XGBoost (XGB).

The contributions of this research include to:
1. identify suitable imputation technique to handle missing data problems on IoT sensor network deployed for the quantification of GHGs.

2. develop a strategy based on feature selection and data imputation to support on-site sensor calibration

3. present reliable technique based on supervised regression methods for data quality improvement of LCS in environmental monitoring networks.

In section 2 , we present the motivation for this work while section 3 details the current state of the art with respect to imputation and sensor calibration. A detailed description of the dataset is presented in section 4 while section 5 described the imputation methods used, the sensor calibration process is described in section 6. Section 7 contains the results of the analysis and section 8 has the conclusion and recommendations for future work.

\section{Motivation}

Inspired by the numerous successes of modern machine learning processes, particularly in the development of imputation algorithms which have been found useful for handling missing values in areas such as medical data[21], sentence generation[22], image concealment [23] and data compression[24]. We explore the possibility of applying imputation techniques for imputing missing data in LCS networks in environmental monitoring research. Due to the nature of LCS, they are often challenged by the problems of missing data and this hinders the application of advanced analysis on the data collected by these sensors. Most researchers in this domain resort to deleting the cases with missing values when applying the dataset for further analysis. This method is ineffective as it simply ignores the cases with missing data, and does not take into consideration the complex distribution in environmental data thereby leading to imprecision and bias. Imputation is capable of learning missing data either as a single value or as multiple possible values to address uncertainties. In cases where data distribution is of interest, imputation can estimate the most probable distribution of the data rather than estimating the unobserved data[25]. In this study, we evaluate the potentials of imputation in LCS dataset and the implication of data imputation for on-site sensor calibration.

\section{State of the art}

Missing or inconsistent data have been a major issue in data analysis since the origin of data col- 
lection. Methods for handling missing data ranges from the naive deletion of instances with missing values to modern machine learning imputation techniques. The suitability of an imputation method can be influenced by the missingness mechanism. Three different missing data mechanisms exist and these mechanisms can affect the accuracy of an imputation method. Techniques for handling missing values are generally assessed based on the three missingness mechanism: Missing Completely at Random (MCAR) where the missingness occur completely at random with no dependency on any of the variables i.e. the distribution of missingness is independent on either the observed values or the missing values. Missing at Random (MAR) where the missingness depends only on the observed values but not on the missing values. Missing Not at Random (MNAR) where the missingness depends both on the observed and missing values [26]. Hedge et al. compared the performance of Probabilistic Principal Component Analysis (PPCA) and MICE for the imputation of missing data in healthcare dataset[27]. Their analysis began with a complete baseline dataset which included medical and dental variables, simulating missing data and its imputation assuming that values were MCAR. Their work showed PPCA to outperform MICE for this purpose. Stekhoven et al. proposed an iterative imputation strategy based on random forest (missForest) by averaging over several unpruned classification or regression trees[14]. They performed their analysis on multiple datasets from a diverse selection of biological fields with artificially introduced missing values at different rates. Their work showed missForest is able to handle missing data on dataset consisting of different data types including continuous and categorical data. Comparatively analysing missForest with other imputation methods such as KNN, they showed missForest to outperform the other methods especially in data settings where complex interactions and non-linear relationships were suspected. Penone et al. evaluated the performance of four imputation methods including KNN, missForest, MICE and Phylopars for estimating missing data in trait datasets[28]. They tested possiblity of imputed datasets retaining underlying allometric relationship among traits. Their analysis were performed on a trait dataset of the mammalian order carnivora. In their work, they also evaluated whether including phylogenetic information which is a required input for phylopers improved the performance of KNN, MICE and missForest. They also evaluated the extent to which the allometric relationship between two traits; body mass and longevity were conserved for imputed dataset. Hariz et al. suggested the combination of different imputation methods for imputing missing continuous data rather than using individual imputation techniques[29].

In the environmental monitoring field where LCS are usually employed to supplement existing traditional monitoring solutions, data quality assurance is essential for the sensors and can be achieved through frequent calibration of the sensors. However, previous studies in the IoT sensor calibration domain have drawn conclusions based on the assumption of a complete dataset[30, 31]. The topic of missing data has attracted little or no attention in IoT sensor calibration space. Missing data reduces the representativeness of samples, thereby reducing the statistical power of a study which in turn produces bias estimates and can lead to invalid conclusions[32]. This distortion can adversely affect sensor calibration processes.

Calibrating LCS on imputed dataset rather than on a dataset where incomplete records have been discarded can help to yield more accurate calibration result, improving on-site sensor performance and ensuring that the sensors are collecting accurate data.

In this study, we examine the effect of missing and imputed data on LCS calibration. We exploit the techniques proposed in literature to impute missing LCS data and subsequently use the imputed dataset for sensor calibration. We then evaluate the performance of sensor calibration by different supervised regression models trained on the different imputed datasets.

Previous studies have proposed different methods for sensor calibration. Spinnelle et al. applied linear/multi linear regression and supervised machine learning techniques for the calibration of a cluster of low-cost $\mathrm{O}_{3}, \mathrm{NO}_{2}, \mathrm{NO}, \mathrm{CO}$ and $\mathrm{CO}_{2}$ sensors over a two week calibration period. Based on the measurement uncertainty estimated by orthogonal regressions of sensors and reference data, their work showed ANN to be a suitable calibration model for the sensor clusters while both simple and multiple linear regressions provided high level of measurement uncertainties[33, 34]. De Vito et.al proposed and evaluated the calibration of low cost gas multi-sensor devices in an urban air pollution monitoring mesh using neural networks and a two week on-site recorded data for benzene, $\mathrm{CO}, \mathrm{NO}_{2}$ and $\mathrm{NO}_{\mathrm{x}}$ pollutants quantification. Their work showed the feasibility of obtaining a neural calibration capable of allowing multi sensor devices to successfully operate on the field carrying out pollutant estimation with optimal result even for a limited number of training periods[35, 36]. Okafor et.al applied simple/multi linear regression and artificial neural network for the calibration of low-cost $\mathrm{O}_{3}$ and $\mathrm{NO}_{2}$ sensor devices. Their work evaluated the performance of different feature selection techniques in identifying factors that affect on-site sensor measure- 
ments and applied data fusion to exploit the correlation existing between similar sensors. Experimental results from their work showed that linear regressions and neural network are able to minimize estimation errors from the sensors with respect to conventional station outputs[37, 38].

\section{Dataset Description}

This study utilized publicly available datasets of Ozone $\left(\mathrm{O}_{3}\right)$ and combined Nitrogen dioxide and Ozone $\left(\mathrm{NO}_{2} / \mathrm{O}_{3}\right)$ measurements from Aeroqual and CairclipNO $\mathrm{O}_{2} / \mathrm{O}_{3}$ sensors deployed in triplicates in collocation with Federal Equivalent Methods (FEM) Monitors at an urban regulatory site in Denver Colorado, United States of America, over a six months monitoring period[39]. Temperature (T) and Relative Humidity (RH) sensors were also available on the site. All sensors and FEM monitors logged data per minute in part per billion (ppb) from 2015-09-08 23:00:00 to 2016-02-22 08:49:00. Table 1 contains information about the sensors, manufacturers and operating mechanism.

\begin{tabular}{|c|c|c|c|}
\hline Manufacturer & Sensor & Operating mechanism & Buantity \\
\hline SM50/Aeroqual (New Zealand) & $\mathrm{O}_{3}$ & Electrochemical sensors & 3 \\
\hline Cairclip/Cairpol (France) & NO2 and O3 combined (ppb) & Electrochemical sensors & 3 \\
\hline
\end{tabular}

Table 1: Selected sensors

The sensors are commercially available, low cost and with relatively high market prevalence. More information about the sensors and the dataset can be found in [39]. The variance of the samples split into hourly slots was low, hence we averaged the per minute measurements into hourly measurements by taking the mean of the samples for each hour of recorded data and a total of 3994 samples were obtained. $16.7 \%$ and $29.2 \%$ of values were initially missing from the $\mathrm{O}_{3}$ and $\mathrm{NO}_{2} / \mathrm{O}_{3}$ datasets respectively.

Table 2 shows the number and percentage of observed, missing and combined (observed+missing) values on each variable on the $\mathrm{O}_{3}$ dataset. We did not include that of the $\mathrm{NO} 2 / \mathrm{O} 3$ dataset here due to space limitation.

The sensors exhibited varying correlation with the reference instruments. Hourly- averaged $\mathrm{O}_{3}$ Pearson correlation of the sensors and reference measurement is $>0.9$ as shown in figure 1 while that of $\mathrm{NO}_{2} / \mathrm{O}_{3}$ sensors to $\mathrm{NO}_{2} / \mathrm{O}_{3}$ reference ranges between 0.32 to 0.57

To asses the performance of imputation methods, additional missing values were artificially introduced to the datasets randomly (as illustrated on table 3) and at consecutive periods (as per table 4). Imputation was performed using the various imputation techniques and the imputation accuracy was calculated. The imputation accuracy is defined by the Root Mean Squared Error (RMSE) between imputed and original values values.

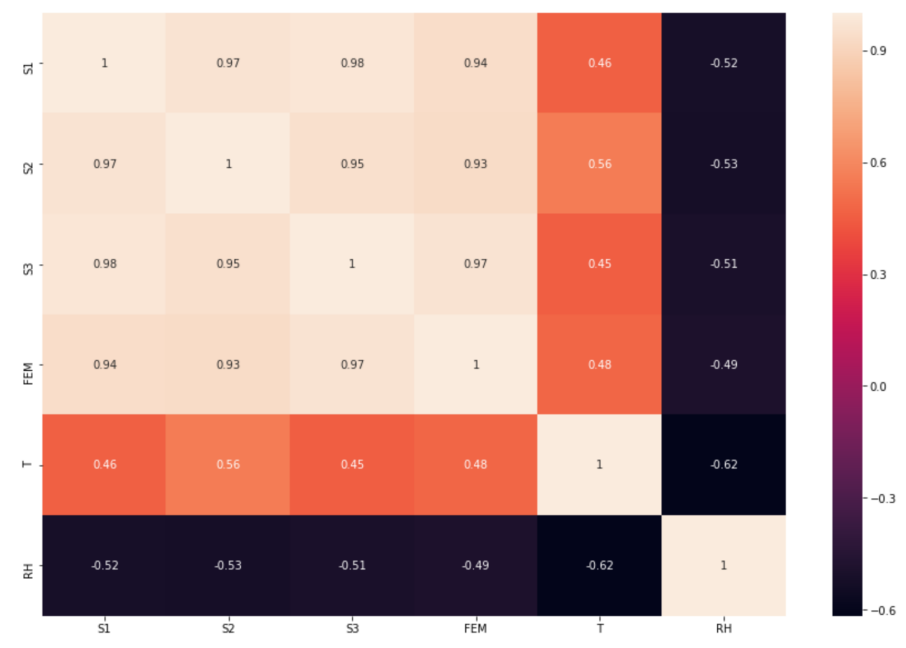

Figure 1: Correlation coefficients of sensors and reference measurements

\section{Methodology}

We employed multiple imputation techniques; MICE, missForest and KNN to impute missing values on the IoT sensors datasets. Our approach involves an initial evaluation of the correlation between features. Features exhibiting high correlation with the variable(s) to be imputed were then selected and included in the imputation model. The heatmap in figure 1 shows the correlation between variables. The $\mathrm{O}_{3}$ sensors (S1, S2, S3) showed high correlation among sensors and with the FEM monitor, the sensors also exhibited varying positive correlation with $\mathrm{T}$ and negative correlation with $\mathrm{RH}$.

We introduced artificial missingness following the patterns listed below and examined how the imputation techniques performed on imputation tasks under the different missingness scenarios:

i. missing values on a single sensor unit (variable) with missingness spanning $p$ period of time $p=\{1$ day, 1 wk, 2 wks, 1 month, ... 4 months $\}$

ii. missing values on double sensor units with missingness spanning $p$ period of time $p=\{1$ day, $1 \mathrm{wk}$, 2wks, 1 month, 2 months \}

iii. missing values on all sensor units with missingness spanning a period of upto one month.

iv. missing values on all sensor units with missingness occurring at random points and at different proportion (r) where $r \in\{5 \%, 10 \%, 30 \%, 50 \%, 70 \%\}$. 


\begin{tabular}{|c|c|c|c|c|c|c|}
\hline & \multicolumn{2}{|c|}{ Observed } & \multicolumn{2}{c|}{ Missing } & \multicolumn{2}{c|}{ Combined } \\
\hline Variable & $\mathrm{n}$ & percentage & $\mathrm{n}$ & percentage & $\mathrm{n}$ & percentage \\
S1 & 3325 & 16.5 & 669 & 17.4 & 3994 & 16.7 \\
S2 & 2963 & 14.7 & 1031 & 26.9 & 3994 & 16.7 \\
S3 & 3279 & 16.3 & 715 & 18.6 & 3994 & 16.7 \\
T & 3523 & 17.5 & 471 & 12.3 & 3994 & 16.7 \\
RH & 3523 & 17.5 & 471 & 12.3 & 3994 & 16.7 \\
FEM & 3519 & 17.6 & 474 & 11.89 & 3994 & 16.7 \\
Total & 20132 & 100 & 3832 & 100 & 23964 & 100 \\
\hline
\end{tabular}

Table 2: Comparison of observed and missing values on $\mathrm{O}_{3}$ dataset

\begin{tabular}{|c|c|c|c|c|c|}
\hline$t_{1}$ & $t_{2}$ & $t_{3}$ & $t_{4}$ & $t_{5}$ & $t_{6}$ \\
\hline $\mathrm{X}_{11}$ & $\mathrm{X}_{12}$ & $\mathrm{X}_{13}$ & $\mathrm{X}_{14}$ & $X_{15}$ & $X$ \\
\hline $\mathrm{X}_{21}$ & $\mathrm{x}_{22}$ & $\mathbf{x}_{23}$ & $X$ & $\mathrm{X}_{25}$ & $\mathrm{X}_{26}$ \\
\hline $\mathrm{X}_{31}$ & $\mathrm{X}$ & $\mathbf{x}_{33}$ & $\mathrm{x}_{34}$ & $\mathbf{X}_{35}$ & $\mathrm{X}_{36}$ \\
\hline $\mathrm{X}_{41}$ & $\mathrm{x}_{42}$ & $\mathbf{x}_{43}$ & $\mathrm{X}_{44}$ & $\mathrm{X}_{45}$ & $X$ \\
\hline $\mathrm{X}_{51}$ & $\mathrm{X}_{52}$ & $X_{53}$ & $\mathrm{X}_{54}$ & $\mathrm{X}$ & $\mathrm{X}_{56}$ \\
\hline
\end{tabular}

Table 3: Missing data pattern:missing values occurred at random points ( $\mathrm{t}=$ time)

\begin{tabular}{|c|c|c|c|c|c|}
$\mathrm{t}_{1}$ & $\mathrm{t}_{2}$ & $\mathrm{t}_{3}$ & $\mathrm{t}_{4}$ & $\mathrm{t}_{5}$ & $\mathrm{t}_{6}$ \\
\hline $\mathrm{x}_{11}$ & $\mathrm{x}_{12}$ & $\mathrm{x}_{13}$ & $\mathrm{x}_{14}$ & $\mathrm{x}_{15}$ & $\mathrm{x}$ \\
\hline $\mathrm{x}_{21}$ & $\mathrm{x}_{22}$ & $\mathrm{x}_{23}$ & $\mathrm{x}_{2}$ & $\mathrm{x}_{25}$ & $\mathrm{x}_{26}$ \\
\hline $\mathrm{x}_{31}$ & $\mathrm{x}_{33}$ & $\mathrm{x}_{33}$ & $\mathrm{x}_{34}$ & $\mathrm{x}_{35}$ & $\mathrm{x}_{36}$ \\
\hline $\mathrm{x}_{41}$ & $\mathrm{x}_{42}$ & $\mathrm{x}_{43}$ & $\mathrm{x}_{44}$ & $\mathrm{x}_{45}$ & $\mathrm{x}$ \\
\hline $\mathrm{x}_{51}$ & $\mathrm{x}_{52}$ & $\mathrm{x}_{53}$ & $\mathrm{x}_{54}$ & $\mathrm{x}$ & $\mathrm{x}_{56}$ \\
\hline
\end{tabular}

Table 4: Missing data pattern: missing values at consecutive periods

To the best of our knowledge, this is the first study that have considered the performance of Multiple Imputation (MI) strategies on IoT sensors measuring GHGs concentrations, with missing values occurring over consecutive periods.

\subsection{Multiple Imputation}

As opposed to Single Imputation (SI), MI supports analysis that makes use of all possible information on a dataset[40]. MI accounts for the statistical uncertainties in imputations and also yields more accurate results. They involve filling in the missing values multiple times, creating multiple complete datasets. MI techniques were developed to handle uncertainties in imputation beyond what SI can carter for. They are able to recover information which would otherwise be lost when observations with missing values are excluded in the analysis, thus, helping to minimize bias.

The validity of analysis relying on imputed data would however depend on the correct specification of the imputation model, a common question when dealing with missing values is what proportion of missingness is acceptable before inference with MI becomes valid. Previous studies have identified various upper and lower limits, observing also that the availability of other auxiliary variables which can predict the missingness and/or are associated with the missing values may be an important consideration[41]. The inclusion of auxiliary variables in the imputation model can yield unbiased estimates even at high proportion of missing values (upto 90\%) [41].

Correctly specifying the imputation model is necessary for obtaining accurate analysis with the imputed dataset. The imputation model and the analysis model should be as compatible as possible[41]. This is particularly useful in in-situ network of IoT sensors where imputed dataset may be applied in sensor calibration to ensure accurate measurements. It is useful to include in the imputation model variables that would be used in the calibration process i.e the target variable and other auxiliary variables such as temperature and relative humidity. These variables may exhibit significant relationship with the variable(s) containing missing values and as such, capable of predicting the missing values, therefore including these variables will help to reduce bias on the imputed dataset and also help to improve the precision of the final estimates.

\subsection{Multiple Imputation by Chain Equation (MICE)}

MICE which is sometimes called "fully conditional specification" or "sequential regression multiple imputation" is an MI strategy which has proved useful in addressing missing values. The MICE imputation strategy is flexible and can be used in a wide range of settings. The chain equation approach in MICE can 
handle variables of different types. e.g continuous, categorical and binary variables. MICE assumes that the missing values on the variables are $M A R$, implying that the probability that a value is missing depends only on the observed values and not on the missing values. The MICE method imputes data on a variable by variable basis by specifying an imputation model per variable. We briefly outline below, the MICE procedure used in this study:

1. mean value imputation (a single imputation process) was first carried out for every missing value on the dataset (this mean imputation could be regarded as a "place holder").

2. the "place holder" created in(1) for one of the variables (i.e. output from one of the sensors $\left(\mathrm{S}_{\mathrm{j}}\right)$ were set back to missing.

3. the observed values from $S_{j}$ in step 2 were linearly regressed against the other variables on the imputation model. $S_{j}$ being the dependent variable and the other variables being the independent variables on the regression model.

4. The missing values in $\mathrm{S}_{\mathrm{j}}$ were then replaced by predictions from the regression model.

5. Step 2-4 were repeated for each variable with missing values.

A complete cycle for each variable constitute one iteration. At the end of one cycle, all of the missing data were replaced with predicted values from the regression, with the predictions reflecting the relationships observed on the dataset.

The entire process of iterating through all the variables were repeated until convergence, at the end, the final imputations were retained, this final set of imputed values and the observed values resulted in one complete dataset.

It is essential to know the number of imputation rounds necessary for a good statistical inference. The authors in [42] suggested that small value of imputation rounds (n) on the order of 3 to 5 yields an excellent result. Schafer et.al stated that not more than 10 imputations rounds are usually required[43], however, Graham et.al after using a monte Carlo simulation to test multiple imputation models across several scenarios in which the fraction of missing information for the parameter being estimated and $n$ were varied, recommended that many more imputation rounds than previously considered sufficient should be performed[44] .

We tested the effects of imputation rounds on both the $\mathrm{O}_{3}$ and $\mathrm{NO}_{2} / \mathrm{O}_{3}$ datasets, setting the imputation rounds to $n$ where $n \in\{5,10,30\}$. The performances of the imputation rounds based on RMSE between imputed and original values for different duration of missingness are presented in Table 5 and discussed on the results section of this work.

\subsection{Missforest}

missForest is an iterative imputation method that is based on Random Forest (RF). In previous literature, missForest was identified to exhibit attractive computational efficiency and capable of handling missing value imputation on high dimensional datasets[14]. In this study, we evaluate the performance of missForest in a comparative analysis with MICE and KNN strategies for handling missing values on IoT sensor network. An iterative imputer scheme was used by first training an $\mathrm{RF}$ on the observed values, followed by predicting the missing values and then proceeding iteratively. The RF algorithm has an in-built function capable of handling missing data by weighing the frequency of the observed values on a variable with the RF proximities after being trained on the initially mean imputed dataset. This method requires the availability of complete dataset for training the forest. We however adopted an approach which involved training an RF on the observed data, similar to the method proposed in [14].

For any variable $S_{j}$ containing missing values at points $i_{\text {missing }}$, where $i_{\text {missing }} \in\{1 \ldots n\}$ the dataset was separated into 4 parts:

\section{The observed values of $S_{j}$ denoted by $y_{\text {observed }}$}

\section{The missing values of $\mathrm{S}_{\mathrm{j}}$ denoted by $\mathrm{y}_{\text {missing }}$}

3. The other variables contained on the dataset (other than $S_{j}$ with observations at point $\mathrm{i}_{\text {observed }}=\{1, \ldots \mathrm{n}\}$ denoted by $\mathrm{X}_{\text {observed }}$

4. The other variables contained on the dataset (other than $\mathrm{S}_{\mathrm{j}}$ ) with observations $\mathrm{i}_{\text {missing }}$ denoted by $\mathrm{X}_{\text {missing }}$

The iterative Imputer algorithm began by making an initial guess for the missing values in $\mathrm{S}$ using mean imputation. The variables $S_{j}, j=1, \ldots p$ are then sorted in accordance with the number of missing values beginning with the variable with the lowest amount of missing values. For each variable $S_{j}$, the missing values are imputed by first fitting an RF with response $\mathrm{y}_{\text {observed }}$ and predictors $\mathrm{X}_{\text {observed }}$. $\mathrm{y}_{\text {missing }}$ is then predicted by applying the trained $\mathrm{RF}$ model to $\mathrm{X}_{\text {missing }}$. The imputation procedure is repeated until a stopping criterion is reached. 


\begin{tabular}{|c|c|c|c|c|c|c|}
\hline & \multicolumn{3}{|c|}{ O3 } & \multicolumn{3}{c|}{ NO2/O3 } \\
\hline Missing period & MICE[5] & MICE[10] & MICE[30] & MICE[5] & MICE[10] & MICE[30] \\
\hline 1 day & $4.98 \times 10^{-5}$ & $4.00 \times 10^{-5}$ & $4.00 \times 10^{-5}$ & $2.69 \times 10^{-1}$ & $2.69 \times 10^{-1}$ & $2.69 \times 10^{-1}$ \\
\hline 1 week & $1.47 \times 10^{-3}$ & $1.46 \times 10^{-3}$ & $1.46 \times 10^{-3}$ & $5.38 \times 10^{-1}$ & $5.38 \times 10^{-1}$ & $5.38 \times 10^{-1}$ \\
\hline 1 month & $2.35 \times 10^{-2}$ & $1.65 \times 10^{-2}$ & $1.60 \times 10^{-2}$ & $7.05 \times 10^{-1}$ & $7.05 \times 10^{-1}$ & $7.05 \times 10^{-1}$ \\
\hline 2 months & $2.95 \times 10^{-2}$ & $2.25 \times 10^{-2}$ & $1.46 \times 10^{-2}$ & $1.07 \times 10^{0}$ & $1.07 \times 10^{0}$ & $1.07 \times 10^{0}$ \\
\hline
\end{tabular}

Table 5: RMSE of MICE imputation rounds at different duration of missingness on $\mathrm{O}_{3}$ and $\mathrm{NO}_{2} / \mathrm{O}_{3}$ datasets

\subsection{K-Nearest Neighbor}

Inspired by the work in [45], we employed KNN to handle missing values on IoT sensors datasets. For each variable on the dataset, the missing values were imputed by finding the $\mathrm{k}$ non-missing values on the sample which are closest to the missing data point. The average of these $\mathrm{k}$ closest values are then taken and used to fill in the missing point. We determined the closest neighbours to the missing point by using an euclidean distance metric given by equation 1 to calculate the distance between the missing point $(\mathrm{x})$ and the non missing neighbour $(\mathrm{y})$.

$$
\sqrt{\sum_{i=1}^{k}\left(x_{\mathrm{i}}-y_{\mathrm{i}}\right)^{2}}
$$

Euclidean distance remains the most obvious way for representing the distance between two points. It measures the length of a segment connecting the two points together. We set $\mathrm{k}=3$ and used the average of these 3 nearest neighbours to the missing point to fill the missing point. Due to the fact that KNN depends on the distance between samples, the scale of the predictor variables can significantly influence the distance among samples, for this reason, we centred and scaled all predictor variables before applying KNN so as to avoid this potential bias and also to enable each predictor to contribute equally to the distance calculation.

\section{Sensor calibration using imputed datasets}

Measurements from LCS are usually unreliable and often result in unsatisfactory level of accuracy. To meet measurement accuracy and to ensure that data quality of LCS is sufficient, frequent calibration and data validation are essential. Calibrating sensors however, requires the availability of complete data points for sensors and reference measurements.

We experiment the feasibility of LCS calibration using imputed dataset and compared the performance to Complete Case Analysis (CCA); a situation which involves the complete elimination of missing data points from the dataset. The FEM monitor provided the reference data for the calibration process and we relied on the predictive variables from different sensors as input, this approach which was described in previous study as sensor fusion have been identified to provide more consistent, reliable and accurate results[37].

Meteorological factors such as Temperature (T) and Relative Humidity (RH) can affect LCS outputs. Figure 2 shows the scatter plot of sensors and reference data color-coded with meteorological factors, indicating the effects of both $\mathrm{T}$ and $\mathrm{RH}$ on $\mathrm{O}_{3}$ and $\mathrm{NO}_{2} / \mathrm{O}_{3}$ measurements. At low $\mathrm{T}$, the $\mathrm{O}_{3}$ and $\mathrm{NO}_{2} / \mathrm{O}_{3}$ values measured by the sensors were lower than that of the reference monitors but as $T$ increases, the sensors values tend to be larger than that of the reference monitor showing a positive interference of $\mathrm{T}$ with sensor measurements. Also, $\mathrm{O}_{3}$ sensor values tend to be higher at lower RH.

To ensure collection of accurate data by LCS, it is important to account for the effects of meteorological factors on sensors output, hence, factors affecting LCS outputs should be identified and included in the calibration model.

All data handling and processing in this study were performed using python 3 on a jupyter notebook which is included as part of the Anaconda distribution[46].

Initial exploratory data analysis on the datasets showed significant correlation existing between the $\mathrm{O}_{3}$ sensors and FEM monitor data, the $\mathrm{NO}_{2} / \mathrm{O}_{3}$ data showed low to moderate correlation with $\mathrm{NO}_{2} / \mathrm{O}_{3} \mathrm{FEM}$ monitor data. To improve the data quality of the sensors to a standard comparable to high grade monitors, their data was treated through the process of calibration using different machine learning regression methods. After imputation of the missing values using the different techniques described in section 5, the variables were analysed for multicollinearity using variance inflation factor (VIF) tool from the statsmodels package in python. Multicollinearity among independent variables will result in less reliable statistical inference, hence variables exhibiting high linear relationships with other variables as well as those exhibiting low level of significance were eliminated through a 


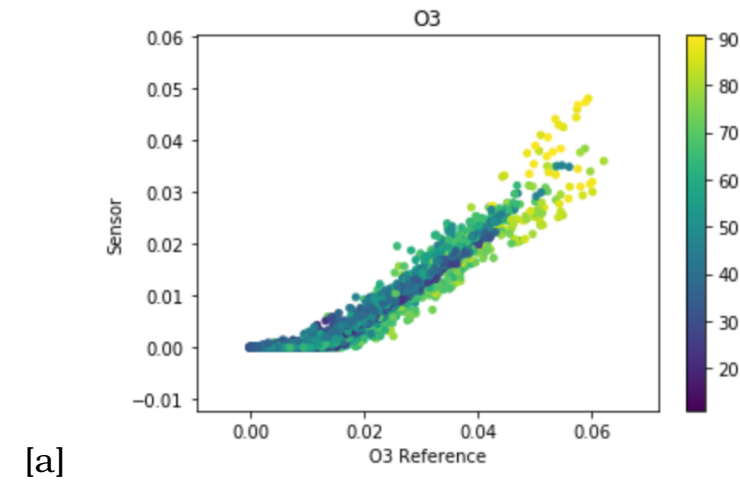

[a]

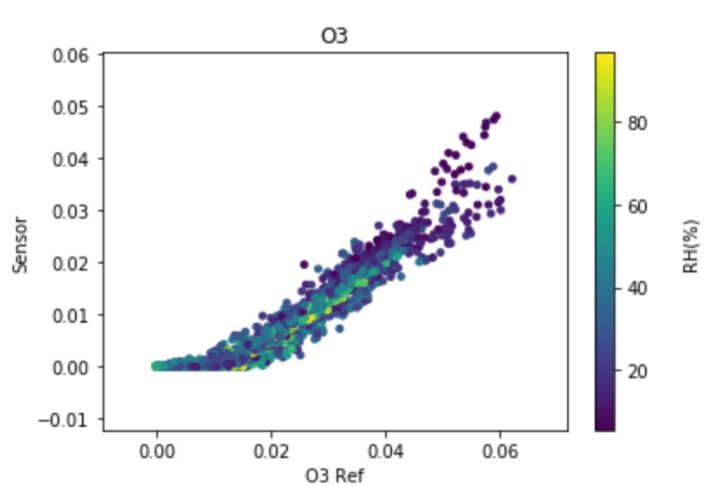

[c]

Figure 2: Scatter plots of sensor and reference data, color-coded with meteorological factors: (a-b) temperature, (c-d) relative humidity
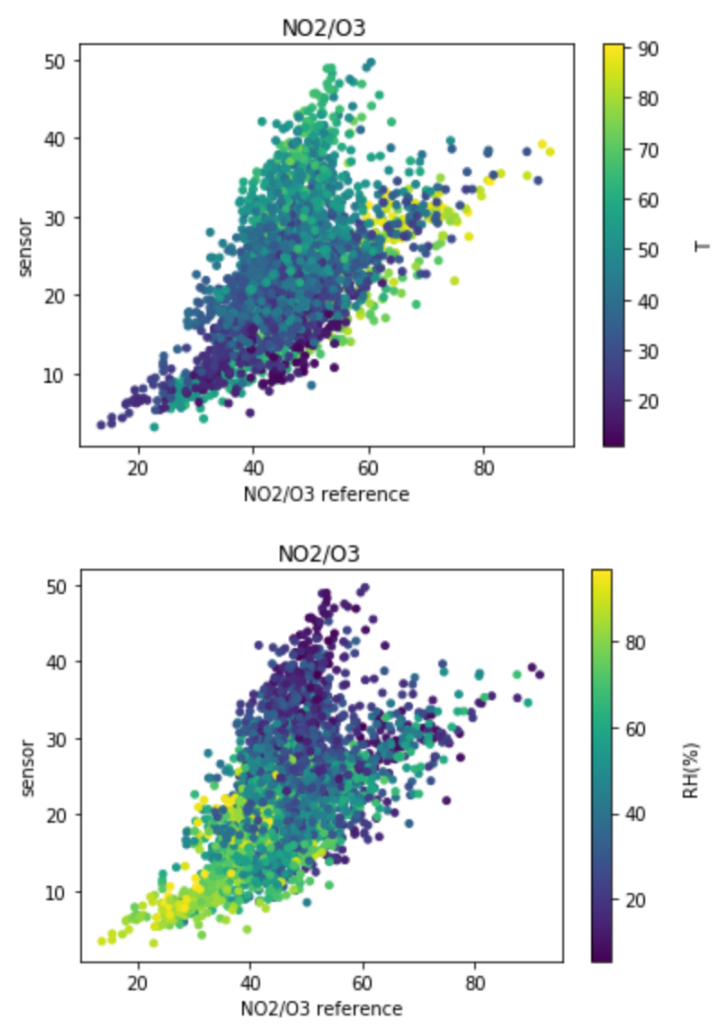

[d] backward elimination process and were not included in the calibration model.

\subsection{Multiple Linear Regression (MLR)}

A MLR calibration model was used to fit the explanatory variables to the FEM monitor data as shown in equation 2.

$$
y_{\text {Ref }}=\beta_{0}+\beta_{1} S_{1}+\beta_{2} S_{2}+\beta_{3} S_{3}+\beta_{4} T+\beta_{5} R H
$$

As per Cordero et.al, MLR is a suitable model for electrochemical sensors because their response to gas concentrations is linear and the cross- sensitivities are additive[47]. MLR has previously been applied in the calibration of low cost air quality sensors[47][48].

\subsection{Decision Tree (DT)}

Decision Tree (DT) provides non-parametric method for partitioning dataset. It can be used to solve both regression and classification problems. The technique can aid the description, generalization and categorization of a given set of data by breaking the dataset into smaller subsets while incrementally developing an associated decision tree with decision nodes and leaf nodes. We used Grid search to get the best set of hyperparameters for the model, we tested different values for the min sample split $s=[5,10,15,20]$ and $s=10$ was found to be the best for the model with max depth of 3. A 10-fold cross validation was used to estimate the performance of the model.

\subsection{Random Forest (RF)}

$\mathrm{RF}$ works by constructing an ensemble of decision trees through a bootstrap aggregation technique. This process involves training each DT on different data samples where sampling is done by replacement. The mean value from the ensemble is then used to predict the value of the new input data. By considering a random subset of the explanatory variables, the root node of the DT is split into sub nodes. The tree is split based on which of the explanatory variable in each random subset is the strongest predictor of the target[49]. The process of node splitting is repeated until a terminal node is reached.

In this experiment, we passed the explanatory variables $\mathrm{X}$ and $\mathrm{y}$ (reference data) to an RF regression calibration model, a grid search cross validation method was used to determine the optimal values of the hyperparameters of the model from a specified range of values, here we choose two hyperparameters max depth and n_estimators to be optimized. we tested max_depths of 3,5,7 and n_estimators[50, 100, $150,200]$ and 3 and 100 were chosen respectively as 
the best performing hyperparameters for the model. As per[50] max_depth is the maximum depth of the tree and n_estimators is the number of trees in the forest. we used a 10-fold cross validation method to determine the model performance, the error metrics Root Mean Squared Error (RMSE), Mean Absolute Error (MAE) (given by equation 3 and 4) and Coefficient of determination $\left(\mathrm{R}^{2}\right)$ were used to evaluate the model performance. The lower the RMSE and MAE, the better and an $\mathrm{R}^{2}$ value closer to 1 indicates good model performance.

$$
\begin{aligned}
R M S E & =\sqrt{1 / n \sum_{i=1}^{n}\left(Y_{\mathrm{i}}-y_{\mathrm{i}}\right)^{2}} \\
M A E & =1 / n \sum_{i=1}^{n}\left(\left|Y_{\mathrm{i}}-y_{\mathrm{i}}\right|\right)
\end{aligned}
$$

(where $n$ is the number of samples, $Y$ is calibrated response and $y$ is target response)

\subsection{XGBoost(XGB)}

In this study, XGBoost was implemented using XGBoost version 0.90 in python. A random search method was used to tune the hyperparameters in the XGboost algorithm. We choose three hyperparameters to tune including n_estimators, n_nodes and learning rate (i.e step size shrinkage). We used early stopping rounds to avoid overfitting on the model.

\subsection{Model evaluation}

Implementation of the regression models was done using the scikit-learn library in python 3. Models performances were evaluated by comparing the calibrated response to reference measurements using the error metrics given in section 6.3. The models were built using imputed dataset and complete case analysis (i.e eliminating missing observations from dataset). The datasets were split into training and test set in proportions of $80 \%$ and $20 \%$ respectively. The training dataset was used for the model training/learning process and the test dataset was used to evaluate the model. The result of the $\mathrm{O}_{3}$ sensor calibration is presented in Table 8 and showed imputed dataset to yield significant result when applied to sensor calibration, outperforming calibration which was conducted using CCA.

\section{Results}

The results and performance evaluation of the different imputation techniques, effect of imputation rounds on MICE imputation as well as effect of imputation on sensor calibration are presented on subsequent sections.

\subsection{Imputation}

The imputation accuracy of the different imputation techniques were compared. The imputation accuracy is defined by the Root Mean Squared Error (RMSE) between the original values and imputed values.

Values were artificially removed from one variable (S1) at specific periods in a consecutive manner. The values were then imputed using MICE, missForest and KNN. Figure 3 shows the performance of the imputation methods. For this case, all the imputation method performed reasonably well with minimal errors $(<0.1)$ even for a long period of missingness (up to 4 months), this could be explained by the availability of other auxiliary variables (i.e other sensors, reference, T and RH measurements) which were included in the imputation model and are able to predict the missingness on S1. MICE (blue bar) however outperformed the rest of the techniques for this task.

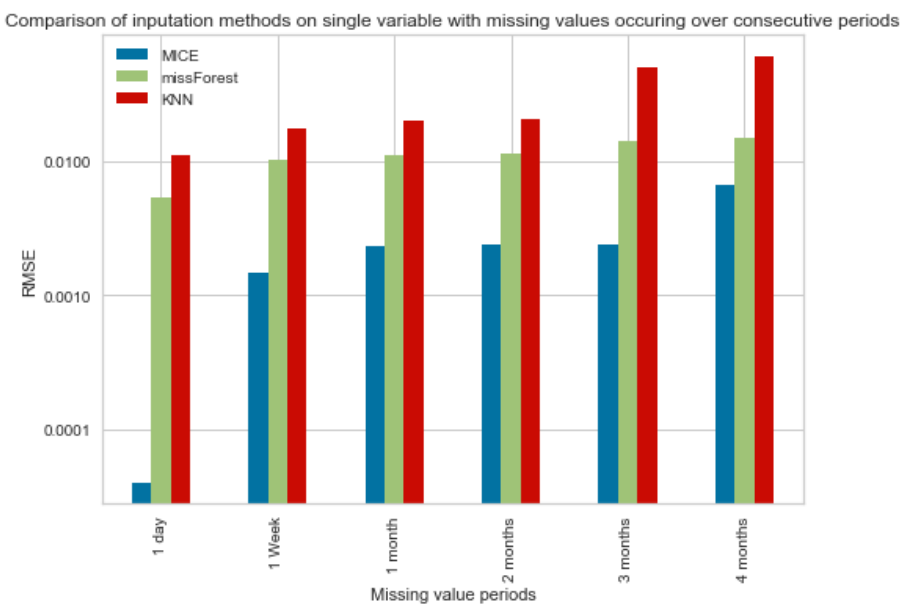

Figure 3: Comparison of imputation methods on single variable with missing values occurring over consecutive periods

Although imputation of missing values on sensor variable(s) over consecutive period of missingness showed promising results, the imputation accuracy, however, tend to decrease over longer periods of missingness as shown in Table 6.

We also evaluated the performance of the strategies for imputation of missing values on two variables, again all methods performed well with minimal RMSE between original and imputed values. While the imputation methods were able to accurately impute the values even for a long period of missing values, the accuracy decreased over longer period of missingness (see Table 6).

The summary statistics of original and imputed dataset for both $\mathrm{S} 1$ and $\mathrm{S} 3 \mathrm{O}_{3}$ sensors are presented in Table 7. The statistics showed good performance of imputation on both $\mathrm{S} 1$ and $\mathrm{S}_{3} \mathrm{O}_{3}$ sensors with mini- 


\begin{tabular}{|c|c|c|c|}
\hline Missing period & MICE & missForest & KNN \\
\hline 1 day & $3.11 \times 10^{-5}$ & $8.07 \times 10^{-5}$ & $5.32 \times 10^{-5}$ \\
\hline 1 week & $1.34 \times 10^{-3}$ & $3.32 \times 10^{-3}$ & $1.55 \times 10^{-3}$ \\
\hline 2 weeks & $1.36 \times 10^{-3}$ & $3.93 \times 10^{-3}$ & $1.49 \times 10^{-3}$ \\
\hline 1 month & $1.65 \times 10^{-2}$ & $1.72 \times 10^{-2}$ & $1.70 \times 10^{-2}$ \\
\hline 2 months & $1.74 \times 10^{-2}$ & $1.76 \times 10^{-2}$ & $1.76 \times 10^{-2}$ \\
\hline
\end{tabular}

Table 6: Performances of MICE, missForest and $\mathrm{KNN}$ for imputation of missing values on two $\mathrm{O}_{3}$ sensor variables over consecutive periods

mal standard deviation of 0.0001 and 0.0005 respectively between observed and imputed values.

Furthermore, we evaluated the performance of the imputation methods when missing values were artificially introduced on all variables over same period of time. Both MICE and KNN had similar performance on this task and both outperformed missForest in this case(see figure 4). Finally, missing values were randomly introduced on the dataset across all variables at different proportion, we ensured that all variable had at least one missing data point and that missing values were distributed across all the variables. missForest outperformed both MICE and KNN in this task as shown on figure 5 .

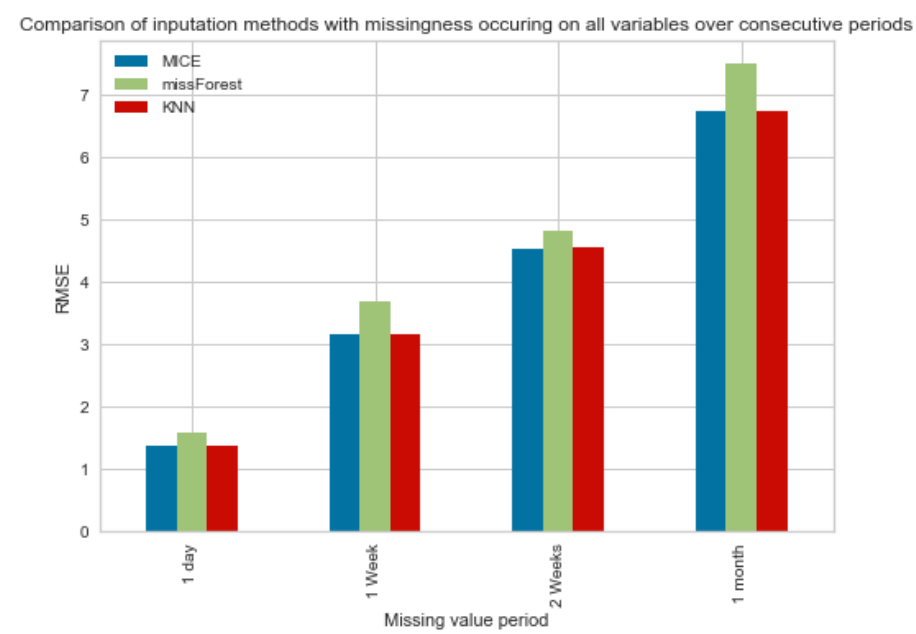

Figure 4: Comparison of imputation methods with missing values occurring on all variables over consecutive periods

\subsection{Imputation rounds}

The result of the imputation rounds for MICE imputation on both the $\mathrm{O}_{3}$ and $\mathrm{NO}_{2} / \mathrm{O}_{3}$ datasets is shown on Table 5. We evaluated the performance of 5,10 and 30 imputation rounds. In terms of RMSE between observed and imputed values on $\mathrm{O}_{3}$ dataset, 10 and 30 imputation rounds maintained same performance at lower proportion of missingness, performing better than 5 imputation rounds. Running the imputation for 30 rounds in the case of longer period of

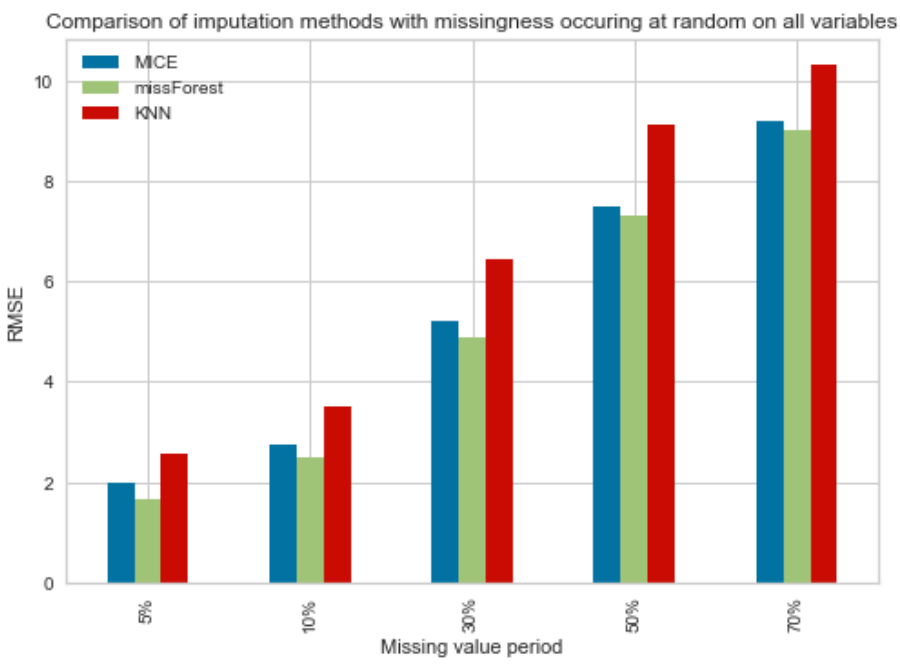

Figure 5: Comparison of imputation methods at different proportion of missingness

missing values ( 1 months and above) however showed more promising performance. Whereas, all 5, 10 and 30 imputation rounds maintained same result even at varying level of missingness in the case of the $\mathrm{NO}_{2} / \mathrm{O}_{3}$ dataset

\subsection{Calibration}

Calibrating the sensors using imputed dataset showed more promising performance when compared to calibration done by deleting missing records from the dataset (i.e Complete case Analysis (CCA). For instance, the MAE existing between uncalibrated/raw $\mathrm{O}_{3}$ sensor and $\mathrm{O}_{3}$ reference outputs is 0.0075. After calibrating the sensor with XGB model trained on missForest imputed dataset, the error was minimized to 0.0011 (see Table 8), this is a reduction of more than $85 \%$ in the MAE compared to $77 \%$ reduction when calibration was done using same XGB calibration model trained on CCA dataset.

\section{Conclusion}

This study explored imputation techniques that could be used to effectively predict missing values on 


\begin{tabular}{|c|c|c|c|c|}
\hline $\mathrm{n}$ & S1(observed) & S1(imputed) & S3(observed) & S3(imputed) \\
min & 3325 & 3994 & 3279 & 3994 \\
max & 0.0000 & -0.0031 & 0.0000 & -0.0031 \\
mean & 0.0481 & 0.0553 & 0.0568 & 0.0647 \\
std & 0.0067 & 0.0075 & 0.0112 & 0.0114 \\
\hline
\end{tabular}

Table 7: Summary statistics of observed and imputed S1 and S3 data with two months of missing values

\begin{tabular}{|c|c|c|c|c|c|c|c|c|}
\hline & \multicolumn{2}{|c|}{ MLR } & \multicolumn{2}{c|}{ DT } & \multicolumn{2}{c|}{ RF } & \multicolumn{2}{c|}{ XGB } \\
\hline & Imputed & CCA & Imputed & CCA & Imputed & CCA & Imputed & CCA \\
RMSE & $\mathbf{0 . 0 0 3 1}$ & 0.0036 & $\mathbf{0 . 0 0 1 8}$ & 0.0036 & $\mathbf{0 . 0 0 1 5}$ & 0.0019 & $\mathbf{0 . 0 0 1 4}$ & 0.0020 \\
MAE & 0.0025 & 0.0289 & 0.0013 & 0.0029 & 0.0011 & 0.0015 & 0.0011 & 0.0017 \\
R2 & $\mathbf{0 . 9 4 2 5}$ & 0.9343 & $\mathbf{0 . 9 8 1 8}$ & 0.9117 & $\mathbf{0 . 9 8 6 7}$ & 0.9449 & $\mathbf{0 . 9 8 8 1}$ & 0.9494 \\
\hline
\end{tabular}

Table 8: Comparison of imputation and complete case analysis on O3 sensor calibration models

the datasets of LCS deployed for the quantification of GHGs . Result of the analysis identified missforest imputation method to be more efficient for imputing missing values when missingness occurred at random points on the dataset even for high proportion of missing values. MICE outperformed both KNN and missForest when missingness occurred consecutively over specific period of time on single or double sensor variables. Furthermore, the imputed datasets were employed in sensor calibration to ascertain any impact imputed data may have on sensor calibration compared to complete case analysis ( a situation which involves discarding missing data records from the dataset before applying calibration). The performance of different calibration models built using different supervised regression algorithms but trained on the different imputed datsets were evaluated. The analysis showed that applying imputation to handle missing values on LCS before calibration improved the performance of the sensors, reducing the MAE existing between raw sensor outputs and gold standard (FEM monitor outputs) by more than $85 \%$.

Due to time and resource constraint, this research has focused on dataset from a limited number of electrochemical gas sensors ( 3 units each of $\mathrm{O}_{3}$ and $\mathrm{NO}_{2} / \mathrm{O}_{3}$ sensors), future research direction can focus on other type of gas sensors such as Non-Dispersive Infrared (NDIR) sensors. While this study has been able to show the effectiveness of data imputation on missing LCS values and the importance of same on sensor calibration, further research is required to ascertain the maximum period of time upon which multiple sensors can have missing values for imputation to be valid and applicable for use in sensor calibration tasks.

\section{Acknowledgements}

This work is supported by Schlumberger Foundation through the Faculty for the Future programme, Tertiary Education Trust Fund (TETFUND-Nigeria) and the SmartBOG project under EPA Research Programme 2014-2020 42617/03

\section{References}

[1] MacFaul, L. 'Monitoring greenhouse gases', Verification Yearbook 2004, VERTIC, London.

[2] J. Loy-Benitez, S. Heo and C. Yoo, "Imputing missing indoor air quality data via variational convolutional autoencoders: Implications for ventilation management of subway metro systems", Building and Environment, vol. 182, p. 107135, 2020. Available: 10.1016/j.buildenv.2020.107135.

[3] B. Wells, A. Nowacki, K. Chagin and M. Kattan, "Strategies for Handling Missing Data in Electronic Health Record Derived Data", eGEMs (Generating Evidence and Methods to improve patient outcomes), vol. 1 , no. 3, p. 7, 2013. Available: 10.13063/2327-9214.1035.

[4] K. Ha and K. Kwok, "Dealing with Missing Values in Healthcare Data - BioSymetrics - Drug Discovery Accelerated", BioSymetrics - Drug Discovery Accelerated, 2020. [Online]. Available: https://www.biosymetrics.com/missing-valueshealthcare-data/. [Accessed: 22- Nov- 2020].

[5] K. Sanjar, O. Bekhzod, J. Kim, A. Paul and J. Kim, "Missing Data Imputation for Geolocationbased Price Prediction Using KNN-MCF 
Method", ISPRS International Journal of GeoInformation, vol. 9, no. 4, p. 227, 2020. Available: 10.3390/ijgi9040227.

[6] Y. Chen, Y. Lv and F. Wang, "Traffic Flow Imputation Using Parallel Data and Generative Adversarial Networks", IEEE Transactions on Intelligent Transportation Systems, vol. 21 , no. 4, pp. 1624-1630, 2020. Available: 10.1109/tits.2019.2910295.

[7] K. Lakshminarayan, S. Harp and T. Samad, "Imputation of Missing Data in Industrial Databases", Applied Intelligence, vol. 11, pp. 259-275, 2020. [Accessed 22 November 2020].

[8] L. Ehrlinger, T. Grubinger, B. Varga, M. Pichler, T. Natschläger and J. Zeindl, "Treating Missing Data in Industrial Data Analytics," 2018 Thirteenth International Conference on Digital Information Management (ICDIM), Berlin, Germany, 2018, pp. 148-155, doi: 10.1109/ICDIM.2018.8846984.

[9] "Air Sensor Guidebook - Science Inventory - US EPA", Cfpub.epa.gov, 2020. [Online]. Available: https://cfpub.epa.gov/si/si_public_record_report [Accessed: 16- Dec- 2020].

[10] M. S. Osman, A. M. Abu-Mahfouz and P. R. Page, "A survey on data imputation techniques: water distribution system as a use case," IEEE Access, vol. 6, pp. 63279-63291, Jun. 2017.

[11] John W Graham. Missing data analysis: Making it work in the real world. Annual review of psychology, 60:549-576, 2009.

[12] M. P. Gómez-Carracedo, J. M. Andrade, P. LópezMahía, S. Muniategui and D. Prada, "A practical comparison of single and multiple imputation methods to handle complex missing data in air quality datasets," Chemom. Intell. Lab. Syst., vol. 134, pp. 23-33, 2014.

[13] M. Azur, E. Stuart, C. Frangakis and P. Leaf, "Multiple imputation by chained equations: what is it and how does it work?", International Journal of Methods in Psychiatric Research, vol. 20, no. 1, pp. 40-49, 2011 . Available: 10.1002/mpr.329.

[14] D. Stekhoven and P. Buhlmann, "MissForestnon-parametric missing value imputation for mixed-type data”, Bioinformatics, vol. 28 , no. 1 , pp. 112-118, 2011. Available: 10.1093/bioinformatics/btr597.
[15] X. Chen, Z. He and L. Sun, "A Bayesian tensor decomposition approach for spatiotemporal traffic data imputation", Transportation Research Part C: Emerging Technologies, vol. 98, pp. 73-84, 2019. Available: 10.1016/j.trc.2018.11.003.

[16] R. Xie, N. Jan, K. Hao, L. Chen and B. Huang, "Supervised Variational Autoencoders for Soft Sensor Modeling With Missing Data", IEEE Transactions on Industrial Informatics, vol. 16, no. 4, pp. 2820-2828, 2020. Available: 10.1109/tii.2019.2951622.

[17] J. Yoon, J.Jordon,M.van der Schaar,"GAIN: Missing Data Imputation using Generative Adversarial Nets", Proceedings of the 35th International Conference on Machine Learning, Stockholm, Sweden,2018.

[18] D. Snow, "MTSS-GAN: Multivariate Time Series Simulation Generative Adversarial Networks", SSRN Electronic Journal, 2020. Available: $10.2139 /$ ssrn.3616557

[19] C. Ruffino, R. Hérault, E. Laloy and G. Gasso, "Pixel-wise conditioned generative adversarial networks for image synthesis and completion", Neurocomputing, 2020. Available: 10.1016/j.neucom.2019.11.116.

[20] Q. Wang, H. Fan, G. Sun, W. Ren and Y. Tang, "Recurrent Generative Adversarial Network for Face Completion", IEEE Transactions on Multimedia, pp. 1-1, 2020. Available: 10.1109/tmm.2020.2978633.

[21] A. Purwar and S. Singh, "Hybrid prediction model with missing value imputation for medical data”, Expert Systems with Applications, vol. 42, no. 13, pp. 5621-5631, 2015. Available: 10.1016/j.eswa.2015.02.050.

[22] S.Bowman, L.Vilnis, O.Vinyals, A. Dai, R.Jozefowicz and S.Bengio,"Generating Sentences from a Continuous Space", Proceedings of the 20th SIGNLL Conference on Computational Natural Language Learning (CoNLL), pages 10-21,2016.

[23] J. Koloda, J. Ostergaard, S. Jensen, V. Sanchez and A. Peinado, "Sequential Error Concealment for Video/Images by Sparse Linear Prediction", IEEE Transactions on Multimedia, vol. 15, no. 4, pp. 957-969, 2013. Available: 10.1109/tmm.2013.2238524. 
[24] M. Peralta, P. Jannin, C. Haegelen and J. Baxter, "Data Imputation and Compression For Parkinson's Disease Clinical Questionnaires", Hal.archives-ouvertes.fr, 2021. [Online]. Available: https://hal.archives-ouvertes.fr/hal02570967v1. [Accessed: 06- Jan- 2021].

[25] A. Kazemi and H. Meidani, "IGANI: Iterative Generative Adversarial Networks for Imputation Applied to Prediction of Traffic Data", arXiv:2008.04847

[26] M.Brown, J.Kros, "Data mining and the impact of missing data", Emeralds: Industrial Management and data systems vol 103,pp.611-621, 2003

[27] H. Hegde, N. Shimpi, A. Panny, I. Glurich, P. Christie and A. Acharya, "MICE vs PPCA: Missing data imputation in healthcare", Informatics in Medicine Unlocked, vol. 17, p. 100275, 2019. Available: 10.1016/j.imu.2019.100275.

[28] C. Penone et al., "Imputation of missing data in life-history trait datasets: which approach performs the best?", Methods in Ecology and Evolution, vol. 5, no. 9, pp. 961-970, 2014. Available: 10.1111/2041-210x.12232.

[29] N. Hariz, H.Khoufi and E.Zagrouba, "On combining imputation methods for handling missing values", Lecture Notes in Computer Science (including subseries Lecture Notes in Artificial Intelligence and Lecture Notes in Bioinformatics) (2017)

[30] S. Munir, M. Mayfield, D. Coca, S. Jubb and O. Osammor, "Analysing the performance of lowcost air quality sensors, their drivers, relative benefits and calibration in cities-a case study in Sheffield", Environmental Monitoring and Assessment, vol. 191, no. 2, 2019. Available: 10.1007/s10661-019-7231-8.

[31] K. Yamamoto, T. Togami, N. Yamaguchi and S. Ninomiya, "Machine Learning-Based Calibration of Low-Cost Air Temperature Sensors Using Environmental Data”, Sensors, vol. 17, no. 6, p. 1290, 2017. Available: 10.3390/s17061290.

[32] H. Kang, "The prevention and handling of the missing data", Korean Journal of Anesthesiology, vol. 64, no. 5, p. 402, 2013. Available: 10.4097/kjae.2013.64.5.402.

[33] L. Spinelle, M. Gerboles, M. Villani, M. Aleixandre and F. Bonavitacola, "Field calibration of a cluster of low-cost available sensors for air quality monitoring. Part A: Ozone and nitrogen dioxide", Sensors and Actuators B: Chemical, vol. 215, pp. 249-257, 2015. Available: 10.1016/j.snb.2015.03.031.

[34] L. Spinelle, M. Gerboles, M. Villani, M. Aleixandre and F. Bonavitacola, "Field calibration of a cluster of low-cost commercially available sensors for air quality monitoring. Part B: NO, CO and CO2", Sensors and Actuators B: Chemical, vol. 238, pp. 706-715, 2017. Available: 10.1016/j.snb.2016.07.036.

[35] S. De Vito, E. Massera, M. Piga, L. Martinotto and G. Di Francia, "On field calibration of an electronic nose for benzene estimation in an urban pollution monitoring scenario", Sensors and Actuators B: Chemical, vol. 129, no. 2, pp. 750-757, 2008. Available: 10.1016/j.snb.2007.09.060.

[36] [5]S. De Vito, M. Piga, L. Martinotto and G. Di Francia, "CO, NO2 and NOx urban pollution monitoring with on-field calibrated electronic nose by automatic bayesian regularization", Sensors and Actuators B: Chemical, vol. 143, no. 1, pp. 182-191, 2009. Available: 10.1016/j.snb.2009.08.041.

[37] N. Okafor, Y. Alghorani and D. Delaney, "Improving Data Quality of Low-cost IoT Sensors in Environmental Monitoring Networks Using Data Fusion and Machine Learning Approach", ICT Express, vol. 6, no. 3, pp. 220-228, 2020. Available: 10.1016/j.icte.2020.06.004.

[38] N. Okafor and D. Delaney, "Application of Machine Learning Techniques for the Calibration of Low-cost IoT Sensors in Environmental Monitoring Networks," 2020 IEEE 6th World Forum on Internet of Things (WF-IoT), New Orleans, LA, USA, 2020, pp. 1-3, doi: 10.1109/WFIoT48130.2020.9221246.

[39] W. Jiao et al., "Community Air Sensor Network (CAIRSENSE) project: evaluation of lowcostsensor performance in a suburban environment in the southeastern UnitedStates", Atmospheric Measurement Techniques, vol. 9, no. 11, pp. 5281-5292, 2016. Available: 10.5194/amt-95281-2016.

[40] Bouhlila, D. and Sellaouti, F., 2013. Multiple imputation using chained equations for missing data in TIMSS: a case study. Large-scale Assess-ments in Education, 1(1). 
[41] "A practical guide to multiple imputation of missing data in nephrology", Kidney International. 6-Apr.-2020. [Online]. Available: www.kidneyinternational.org/article/S0085-2538(20)309510/fulltext. [Accessed: 4-Dec.-2020].

[42] Schafer J.L, Olsen M.K, 1998 Multiple imputation for multivariate miss-ing data problems: a data analyst's perspective. Multivariate Behavioral Research 1998, 33: 545-571. $10.1207 / \mathrm{s} 15327906 \mathrm{mbr} 33045$

[43] Schafer J.L 1999. NORM: Multiple imputation of incomplete multivari-ate data under a normal model [computer software]. University Park, PA:Department of Statistics, Pennsylvania State University.

[44] Graham, J., Olchowski, A. and Gilreath, T., 2007. How Many Impu-tations are Really Needed? Some Practical Clarifications of Multiple Imputation Theory. Prevention Science, 8(3), pp.206-213.

[45] "Applied Predictive Modeling", Applied Predictive Modeling, 2020. [Online]. Available: http://appliedpredictivemodeling.com/. [Accessed: 16- Dec- 2020].

[46] "Anaconda - The World's Most Popular Data Science Platform", Anaconda, 2020. [Online]. Available: https://www.anaconda.com/. [Accessed: 17- Dec- 2020].

[47] J. Cordero, R. Borge and A. Narros, "Using statistical methods to carry out in field calibrations of low cost air quality sensors", Sensors and Actuators B: Chemical, vol. 267, pp. 245-254, 2018. Available: 10.1016/j.snb.2018.04.021.

[48] M. Badura, P. Batog, A. Drzeniecka-Osiadacz and P. Modzel, "Regression methods in the calibration of low-cost sensors for ambient particulate matter measurements", SN Applied Sciences, vol. 1, no. 6, 2019. Available: 10.1007/s42452-019-0630-1.

[49] N. Zimmerman et al., "A machine learning calibration model using random forests to improve sensor performance for lower-cost air quality monitoring”, Atmospheric Measurement Techniques, vol. 11 , no. 1 , pp. 291-313, 2018. Available: 10.5194/amt-11-291-2018.

[50] "3.2.4.3.2. sklearn.ensemble.RandomForestRegressorscikit-learn 0.23.2 documentation",Scikitlearn.org, 2020.2 [Online]. Available: https://scikitlearn.org/stable/modules/generated/sklearn.ensemble.RandomForestRegressor.html.[Accessed: 17 Dec 2020]. 